\title{
Hacia nuevos modelos empresariales más sociales y humanos: El papel de las mujeres en procesos de emprendimiento social y economía de comunión
}

\section{Towards New more Social and Human Business \\ Models: The Role of Women in Social and Economy of Communion Entrepreneurship Processes}

\section{PABLO RUIZ PALOMINO}

(Universidad de Castilla La Mancha)

pablo.ruiz@uclm.es

\section{LOUISE KELLY}

(Alliant International University)

kelly@alliant.edu

Resumen: Ante las deficiencias encontradas en el sistema capitalista para reducir desequilibrios sociales y humanos, tanto el emprendimiento social como de economía de comunión se han erigido durante la última década como nuevos modelos empresariales de alta innovación social y humanidad. Sin embargo, el conocimiento sobre este tipo de emprendimiento y las variables que pueden incidir en su activación y desarrollo es todavía escaso. Un caso particular de interés es analizar qué incidencia tiene el sexo del individuo en su proceso de creación, y en concreto, si ser mujer puede actuar como un factor desencadenante. Así, analizando las características propias del emprendimiento social y de economía de comunión, y la literatura sobre mujer emprendedora, mostramos una alta afinidad entre ser mujer y este tipo de emprendimiento, en cuanto a las diferentes fases del proceso de creación de una empresa.

Palabras clave: Emprendimiento Social, Emprendimiento de Economía de Comunión, Creación de Empresas, Innovación Social, Mujeres, Modelos SocioEconómicos Alternativos

\section{JORGE LINUESA LANGREO \\ (Universidad de Castilla La Mancha) \\ jorge.linuesa@uclm.es}

\begin{abstract}
Given the existing deficiencies in the today's capitalist system to reduce social and human imbalances, in recent decades, both social and economy of communion entrepreneurship have emerged as new business models of high social innovation impact and humankind. However, knowledge of this type of enterprise, and the variables that can affect their activation and development is still low. One particular interest is to analyze the impact of sex on the process of starting-up these businesses, and whether being a woman acts as a trigger. Thus, by analyzing the characteristics of social and economy of communion entrepreneurship, as well as utilizing women entrepreneurship literature, we will show compatibilities between being a woman and this type of entrepreneurship, especially with regards to the necessary impetus to successfully address the different stages of the businesses creation process.
\end{abstract}

Keywords: Social Entrepreneurship, Economy of Communion Entrepreneurship, Business Creation, Social Innovation, Women, Alternative Socio-Economic Models. 


\section{INTRODUCCION}

Durante más de cuatro siglos el sistema capitalista —que basa su funcionamiento en el interés propio y el libre mercado ${ }^{1}$ - ha sido la estructura social, económica y política dominante. Este sistema económico propone que la mano invisible del mercado hace posible por sí sola que la suma de los egoísmos individuales repercuta positivamente en toda la sociedad. Sin embargo, aun cuando en el largo plazo es posible que el mercado llegue a cierto nivel de eficiencia socio-económica por sí solo, lo normal es que esta búsqueda continua del interés propio desemboque en avaricia y despreocupación directa por el bienestar y dignidad de los demás. Bajo esta lógica dominante basada en la transacción de intereses y el corto plazo, la persona es considerada como un medio totalmente intercambiable y no como un fin en sí mismo, dejando así espacio a que "el interés propio" impregne las decisiones que se toman a nivel económico-empresarial, con las probables consecuencias negativas que ello puede conllevar sobre los índices de bienestar social y dignidad humana. De hecho, las necesidades más básicas de millones de personas en países tanto industrializados como no industrializados se encuentran aún hoy totalmente insatisfechas; la situación social de estas personas se ve, además, gravemente afectada en momentos de recesión y crisis económico-financiera. En efecto, la última crisis económico-financiera iniciada en 2008 ha llevado a gran parte de la población europea a alcanzar el umbral de la pobreza y a un estado de riesgo de exclusión social ${ }^{2}$. Este valor, si bien tras casi una década ha retrocedido a niveles de pre-crisis, sigue alcanzando todavía hoy a un porcentaje elevado de la población (más del $20 \%)^{3}$, reflejándose así las limitaciones del actual modelo socio-económico por corregir este problema. En España, por ejemplo, los efectos negativos de esta crisis elevaron drásticamente la población en riesgo de pobreza y exclusión social, datos que a día de hoy, y pese a la recuperación macroeconómica mostrada por este país, se han visto, incluso, incrementados ${ }^{4}$.

Ante esta situación, no resulta extraño que de la mano de los sucesivos encuentros anuales celebrados por el Foro Social Mundial se perciba un creciente posicionamiento de la población civil en contra de la validez y eficien-

\footnotetext{
Smith, A. [1776 (2009)].

EUROSTAT (2014).

EUROSTAT (2016).

4 Según EUROSTAT $(2014,2016)$ el índice de Gini, que mide la desigualdad entre los niveles de ingreso en una sociedad, refleja un aumento de dicha desigualad en España: 31,9 en 2007; 34,2 en 2012, y 34,6 en 2016.
} 
cia de las asunciones sobre las que se fundamenta el actual sistema económico de mercado 5 . En dichos Foros, este sistema se ve como incapaz de erradicar problemas sociales indeseables (p.e. pobreza, desigualdades sociales, degradación medioambiental) y se discute la necesidad de dar entrada a nuevos modelos socioeconómicos — de marcado acento social ${ }^{6}$ — que sí puedan abordar de una manera más activa, innovadora y eficiente los problemas y desafíos sociales existentes. Estos nuevos modelos, que dan soporte al Emprendimiento Social $^{7}$ o al Emprendimiento de Economía de Comunión ${ }^{8}$, atraen crecientemente un mayor interés por parte de la sociedad civil, y en especial, por parte de empresarios, inversores y gobernantes 9 . De hecho, la Unión Europea ha trazado recientemente una estrategia de crecimiento, la llamada Estrategia Europa 2020, que incorpora la promoción de proyectos emprendedores sociales $^{10}$. Dichos proyectos son una fuente importante de innovación social ${ }^{11}$, esto es, de respuesta a problemas sociales que representan un gap entre lo que la sociedad necesita y lo que las diferentes instituciones y empresas existentes ofrecen, y que a la vez, es fuente de rendimiento económico.

Desde la aparición de estos nuevos enfoques hasta la actualidad, la literatura académica y profesional ha abordado, abundantemente, el estudio de este nuevo modelo económico-empresarial, tratando de comprender mejor este fenómeno. Uno de los aspectos de análisis que ha cobrado especial interés ha sido el de identificar las principales motivaciones existentes para iniciar un proyecto de este tipo; por lo general, éstas tienen que ver con la necesidad de resolver problemas sociales y contribuir positivamente a la sociedad ${ }^{12}$. No es extraño, entonces, que detrás de estos intereses existan factores relacionados con la personalidad, y que éstos, a su vez, se encuentren marcados por factores personales innatos como la variable "sexo" (hombre/mujer) del emprendedor. De hecho, si bien las tasas de emprendimiento general, que incluye el emprendimiento comercial y social, se caracterizan según los últimos informes del Global Entrepreneurship Monitor (de aquí en adelante, GEM) por un fuerte factor masculino, llegándose a mostrar, incluso, una tasa de 2 a 1 , para los hombres frente a las mujeres en algunas regiones como la europea ${ }^{13}$,

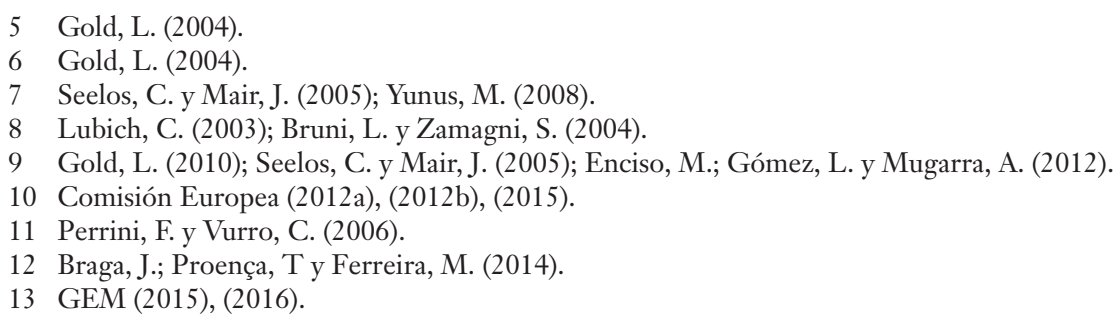


cuando analizamos la actividad empresarial de corte social y no puramente comercial, se muestran mínimas diferencias entre ambos sexos (55\% para hombres y un 45\% para mujeres; GEM, 2015), diferencias que se ajustan todavía más en determinadas regiones del globo (Norte de África, Oriente Medio, Australia y Estados Unidos) ${ }^{14}$. En España, por ejemplo, como reza un estudio liderado por el ESADE, un 53\% de las mujeres emprendedoras prefieren proyectos orientados a generar un impacto social positivo ${ }^{15}$, lo que probablemente se deba a que este colectivo presenta una serie de circunstancias distintivas que empujan más a emprender este tipo de iniciativas. Por ejemplo, los efectos físicos y psicosociales distintos experimentados con la maternidad, los valores sociales (p.e. colaboración, cuidado de otros) y no tan competitivos (éxito, competitividad) experimentados durante la infancia, o las injusticias observadas y/o vividas en términos de salario y "poder" en las empresas tradicionales, pueden ser un estímulo importante a decantarse por un emprendimiento de impacto social positivo ${ }^{16}$. No obstante, y pese a este dato que muestra a la mujer más tendente a desarrollar proyectos emprendedores de una naturaleza social, existe todavía un gap a cubrir en lo que respecta a analizar si la variable "mujer" influye en las diferentes fases de creación de una empresa de este tipo.

El objetivo, por tanto, de este trabajo es analizar cómo afecta la variable "mujer" en las distintas fases y elementos que influyen en la decisión e intención de crear una empresa de marcado acento social. Para ello, este objetivo se desglosa a su vez en tres objetivos más específicos, que conforman las distintas partes de las que se compone este trabajo. En primer lugar, analizaremos cada uno de estos tipos de emprendimiento en términos de operatividad y características compartidas en materia social. Posteriormente, identificaremos los elementos clave en la determinación de la decisión e intención de un individuo por crear una empresa a través del modelo de Krueger y Brazeal ${ }^{17}$. Finalmente, analizaremos conjuntamente las características de la mujer emprendedora ${ }^{18}$ y la de una empresa social ${ }^{19}$ y/o de economía comunión ${ }^{20}$ con el fin de exponer cómo ambos factores se complementan para favorecer la decisión de poner en marcha una empresa de marcado acento social.

\footnotetext{
$14 \operatorname{GEM}(2015)$.

15 WISE (2015).

16 WISE (2015).

17 Krueger, N. y Brazeal, D. (1994).

18 Justo, R. y García, C. (2102).

19 Seelos, C. y Mair, J. (2005).

20 Bruni, L. y Zamagni, S. (2004).
} 


\section{NUEVOS TIEMPOS Y PARADIGMAS EN LA CREACIÓN DE EMPRESAS: HACIA LA INNOVACIÓN SOCIAL}

En los albores de este nuevo siglo XXI se ha podido vislumbrar que el régimen económico que impera, basado en el libre mercado y capitalismo global, si bien ha traído riqueza y bienestar, también es fuente de un número considerable de deficiencias y externalidades negativas. La generación de grandes desigualdades sociales o una fuerte destrucción medioambiental que amenaza la continuidad saludable de la sociedad son algunas de estas deficiencias que parecen generarse bajo dicho modelo socioeconómico. Además, a lo largo de esta última década se han podido vislumbrar los efectos perniciosos que tiene este sistema, en parte, desencadenando graves crisis económico-financieras ${ }^{21}$, de impacto local, pero también global, y en variadas ocasiones, de corte especulativo. $\mathrm{Y}$ es que un sistema caracterizado por mover a los agentes por el interés propio - con ánimo de obtener una mayor riqueza - es un sistema abocado a no dejar lugar directamente a la justicia social. Es verdad que Adam Smith, el propio impulsor del actual sistema económico imperante, promulgaba la necesidad de que las acciones de los diferentes agentes que intervienen en cualquier transacción tomaran como base y guía de sus acciones valores distintos al exclusivo de obtención de la máxima ganancia e interés. En concreto, Adam Smith subrayaba la importancia de ser prudente y moverse por valores tan importantes como la humanidad, justicia, generosidad, el espíritu público, y todo ello, con el fin de obtener el amor, aprobación, gratitud y la confianza de aquellos con quienes se interactuaba ${ }^{22}$. No obstante, todas las acciones previas que se deben desarrollar para conseguir la tan anhelada confianza no se valoran en realidad en términos económicos. El desarrollo de estas acciones por parte de los agentes no aparece reflejado en ningún indicador actual de ganancia financiera, aún cuando estas acciones son estrictamente necesarias para generar confianza, y, por tanto, el intercambio comercial ${ }^{23}$. En efecto, la forma en que el actual sistema económico se encuentra configurado parece desvirtuar la realidad, convirtiendo lo que son transacciones sociales en transacciones meramente comerciales, y guiando la psique humana hacia el desarrollo del homo economicus que sólo busca el máximo interés. En consecuencia, mientras que durante las últimas décadas, el desarrollo de la ciencia ha alcanzado niveles insospechados (biología molecular, ingeniería genética, de-

21 Krugman, P. (2008).

22 Smith, A. [1759 (2004)].

23 Sen, A. (2010). 
sarrollo aeroespacial, etc.), los avances logrados en cuanto a reducción de desigualdades sociales no han sido, desafortunadamente, de gran notoriedad.

Ante este panorama no es extraño que nuevos enfoques socioeconómicos afloren con un claro interés de cambiar de paradigma económico. Así, modelos socioeconómicos como la "economía de comunión" ${ }^{4}$ o el "emprendimiento social" 25 tienen como objetivo resolver problemas sociales y medioambientales derivados de la lógica de funcionamiento del sistema económico actual ${ }^{26}$. Son opciones con un marcado acento en la dimensión social que pretenden conceder más importancia a la persona (y no al interés propio), y considerarla como centro y fin de toda actividad económico-empresarial. Es más, ambos modelos tienen como objetivo principal la finalidad social, más allá de otros objetivos, lo que les convierte en óptimos desarrolladores y precursores de innovación social. En efecto, cuando hablamos de innovación social, hablamos tanto de bienes, servicios, e incluso modelos ${ }^{27}$. Estos deben tener como objetivo principal y explícito el resolver un problema social ${ }^{28}$, y una clara orientación a mejorar el bienestar y desarrollo humano ${ }^{29}$, a través de emplear medios socialmente responsables ${ }^{30}$. Además, dicha innovación debe servir para que el valor creado revierta, sobre todo, en la sociedad — no tanto de forma privada e individualista ${ }^{31}$ — además de actuar bajo el paraguas del principio de justicia social ${ }^{32}$. Así, como veremos a continuación, ambos modelos de emprendimiento, tanto en la raíz como en sus características, se configuran como instrumentos óptimos de innovación orientada a impactar positivamente en la sociedad.

\subsection{El emprendimiento de economía de comunión}

La economía de comunión es un proyecto de desarrollo económico, de carácter solidario, iniciado en 1991 por el movimiento de los Focolares ${ }^{33}$ basado en "la filosofía del dar", diferenciándose así de "la filosofía del tener" que promueve el actual sistema económico imperante. Así, coincidiendo con la es-

\footnotetext{
24 Gold, L. (2004) y (2010).

25 Yunus, M. (2008).

26 Enciso, M.; Gómez, L. y Mugarra, A. (2012).

27 Bureau of European Policy Advisers (2010).

28 Defourny, J. y Nyssens, M. (2008).

29 Pol, E. y Ville, S. (2009).

30 Defourny, J. y Nyssens, M. (2008).

31 Bureau of European Policy Advisers (2010).

32 Martinelli, F. (2012).

33 http://www.focolares.es/es/
} 
piritualidad de este movimiento, que comienza en Italia tras la Segunda Guerra Mundial, este proyecto económico se inicia con la finalidad de que desde las empresas se promueva la comunión dentro de la empresa, entre empresas y con respecto a la más amplia sociedad ${ }^{34}$. El objetivo es enmarcar la lógica racional de la rentabilidad con la "lógica del dar", poniendo en valor a la persona y su autorrealización, a través de la comunión con los demás ${ }^{35}$. Desde las primeras empresas que se desarrollaron en Brasil a inicios de la década de los 90, este tipo de emprendimiento ha crecido y extendido a otras partes del mundo (Argentina, EE.UU., Europa Occidental), principalmente, a través del desarrollo de parques industriales innovadores ${ }^{36}$.

Así, las empresas de comunión presentan un marcado acento social ${ }^{37}$. Estas empresas tienen como principio donar dos terceras partes de los beneficios a contribuir al bien de aquellos más necesitados, además de difundir la cultura del dar y del compartir ${ }^{38}$. Además, estas empresas tienen como objetivo principal no buscar la maximización de beneficios, sino satisfacer a todos los grupos de interés ${ }^{39}$ y lograr un desarrollo sostenible del bien común ${ }^{40}$. Es más, estas empresas mantienen una relación estrecha y un interés en contribuir positivamente a la sociedad ${ }^{41}$, considerando, incluso, a aquellos colectivos en situación de pobreza y exclusión social como grupo de interés principal. En este sentido, tenemos ejemplos reales de empresas de este tipo centradas en la educación infantil y juvenil que benefician con su actividad a familias en riesgo de exclusión $^{42}$. De hecho, este tipo de emprendimiento se caracteriza por distribuir parte de sus beneficios, deliberada y directamente, a la mejora de la situación de aquellos colectivos en situación de pobreza y exclusión social ${ }^{43}$. La tabla 1 muestra los principios de marcado carácter social que comparte este tipo de emprendimiento.

\footnotetext{
34 Bruni, L. (2002); Gold, L. (2004).

35 Gold, L. (2010).

36 Bruni, L. y Zamagni, S. (2004); Gold, L. (2010).

37 Crivelli, L. y Gui, B. (2014); Gold, L. (2004).

38 Bruni, L. y Zamagni, S. (2004).

39 Crivelli, L. y Gui, B. (2014).

40 Grochmal, S. (2016).

41 Gold, L. (2010); Grochmal, S. (2016).

42 Gold, L. (2004)

43 Crivelli, L. y Gui, B. (2014).
} 
Tabla 1. Principios de la empresa de economía de comunión

\section{Emprendedores Trabajadores y Empresa}

- Se adopta la comunión como valor y misión principal de la empresa

- La persona, no el capital, es el centro de la empresa

- Atención especial a aquellos empleados que pasan tiempos difíciles

- Los beneficios se destinan a) a los más necesitados, b) a difundir la cultura del dar, y c) a mejorar la estructura de la empresa, o crear nuevas oportunidades empresariales y laborales

\section{Relaciones con los stakeholders}

- Se emplean los medios más modernos disponibles para ofrecer a los clientes bienes y servicios de calidad a un precio justo

- Los grupos de interés se consideran partners esenciales para la realización del Bien Común

\section{Espiritualidad y Ética}

- Se promueve la persona y su crecimiento personal

- El bienestar de los grupos de interés es siempre evaluado y considerado

- Se respetan las leyes y se trabaja para mejorarlas socialmente

\section{Calidad de Vida, Felicidad y Relaciones}

- La Salud Física y el Bienestar son importantes

- Se evitan las horas excesivas de trabajo

- Se desarrollan productos y servicios medioambientalmente responsables

- La preservación de los Recursos Naturales es una prioridad

- Se emplean instrumentos (reuniones periódicas, momentos de corrección fraterna) que favorecen la comunión dentro de la empresa

\section{Harmonía en el Trabajo}

- Se garantizan las medidas de seguridad, salubridad necesarias para mantener la harmonía relacional y promover la dignidad humana

\section{Formación y Sabiduría}

- Se disponen oportunidades para el aprendizaje continuo y el avance personal y profesional tanto para empleados como externos interesados en el proyecto

\section{Comunicación}

- Se promueve la comunicación abierta y honesta

- Los principios de la Cultura del Dar se promueven y comparten

- Espíritu de apoyo recíproco y solidaridad entre emprendedores de comunión y apertura a agentes externos que deseen contribuir a un mundo más fraterno

Fuente: López, K...; Martínez, Z.L. y Specht, LB. (2013) y Economy of Communion (2016). 


\subsection{El emprendimiento social}

El fenómeno de las empresas sociales es un fenómeno que se remonta a hace ya más de cuatro décadas. Uno de los casos más conocidos, y pioneros de este fenómeno es el caso del Grameen Bank, fundado por Muhamad Yunus en 1976 con el fin de poder conceder crédito (en forma de microcréditos a un bajo y razonable tipo de interés) a agentes socialmente desplazados que no disponían de recursos para salir de su precaria situación social ${ }^{44}$. No obstante, y pese a tener este origen tan remoto, es en las últimas décadas cuando este fenómeno se ha extendido a un ritmo muy rápido ${ }^{45}$. Tal es así que el emprendimiento social ha pasado de desarrollarse únicamente en regiones en desarrollo a países fuertemente industrializados (Unión Europea, Estados Unidos, Japón, Corea del Sur ${ }^{46}$.

El emprendimiento social posee una misión y propósito claramente so$\mathrm{cial}^{47}$. No sólo son empresas que pueden diseñarse para proveer servicio a grupos vulnerables y en situación de riesgo de exclusión social ${ }^{48}$, sino que el empleo de los recursos lo destinan a resolver todo tipo de problemas sociales. Así, y si bien no descuidan el objetivo de la rentabilidad, este tipo de empresas desarrollan su actividad económica con un alto talante de ciudadano socialmente responsable. En este sentido, el emprendedor social sería como un agente de cambio social, caracterizado por: a) adoptar una misión de crear y mantener, principalmente, valor social, b) reconocer y perseguir nuevas oportunidades para cumplir con esa misma misión, implicándose en un proceso de continua innovación, adaptación y aprendizaje, y c) mostrar una gran responsabilidad por las comunidades a las que sirve. En la siguiente tabla (ver Tabla 2) se pueden distinguir la naturaleza y fin social de este tipo de emprendimiento.

\footnotetext{
44 Mair, J. y Shoen, O. (2007).

$45 \operatorname{GEM}(2015)$.

46 Defourny, J. y Nyssens, M. (2008).

47 Dees, J. (2011); Martin, R. y Osberg. S. (2007).

48 Defourny, J. y Nyssens, M. (2008).
} 
Tabla 2. Naturaleza y objetivos de la empresa social

\section{Medioambiente}

- Control de la contaminación

- Restauración o protección del Medioambiente

- Conservación de los recursos Naturales

- Esfuerzos de reciclaje

\section{Energía}

- Conservación de la energía en las operaciones de producción y de marketing

- Esfuerzos para incrementar la eficiencia energética de los productos

- Otros programas de ahorro de energía (p.e. patrocinio del transporte público)

\section{Prácticas justas}

- Empleo y progreso de minorías y mujeres

- Empleo y progreso de personas socialmente desfavorecidas (discapacitados, ex-drogadictos, desempleados de larga duración, etc.)

- Apoyo a empresas propiedad de minorías sociales

\section{Recursos Humanos}

- Promoción de la salud y seguridad laboral

- Desarrollo y formación laboral

- Programas de educación de refuerzo para empleados desaventajados

- Programas de asesoramiento psicológico sobre el uso del alcohol y las drogas

- Asesoramiento sobre la carrera profesional

- Instalaciones de cuidado de niños para padres trabajadores

- Programas de gestión de estrés para empleados

\section{Implicación con la Comunidad}

- Donaciones de dinero, productos, servicios y tiempo de los empleados

- Patrocinio de proyectos de salud pública

- Apoyo a la educación y las artes

- Apoyo a programas recreativos para la comunidad

- Cooperación en proyectos comunitarios (reciclaje, renovación urbana, etc.)

\section{Productos}

- Mejora de la seguridad del producto

- Patrocinio de Programas de Educación sobre seguridad de los productos

- Reducción del potencial contaminante de los productos

- Mejora en el valor nutricional de los productos

- Mejora en el empaquetado y el etiquetado

Fuente: Kuratko, D. F. (2009). 


\section{DECISIÓN E INTENCIÓN DE EMPRENDER: EMPRESAS SOCIALES Y DE ECONOMÍA DE COMUNIÓN COMO FACILITADORAS DEL PROCESO}

\subsection{La intención de crear una empresa: El modelo de Krueger y Brazeal 49}

La identificación y comprensión de los distintos elementos que intervienen en la decisión de emprender es y ha sido objeto de investigación durante décadas $^{50}$. El modelo de Krueger y Brazeal constituye un intento ampliamente aceptado y de gran influencia en esta materia ${ }^{51}$ Dicho modelo se centra en explicar las fases y elementos que explican la decisión de crear una empresa, donde la interacción de factores situacionales y socio-culturales desempeñan un papel importante, y en consecuencia, también las percepciones de deseabilidad y viabilidad de la idea de creación ${ }^{52}$. Además, el modelo de Krueger y Brazeal toma como base la teoría de la conducta planificada de Ajzen ${ }^{53}$, que considera que el comportamiento se predice por las intenciones y éstas, a su vez, por las actitudes hacia el comportamiento (si desarrollar este comportamiento es bueno o malo), la norma subjetiva (lo que dirán personas muy influyentes, cercanas e importantes sobre tal comportamiento) y el control conductual percibido (percepción de tener éxito en desarrollar tal comportamiento). Así, el modelo para predecir la intención emprendedora (ver Figura 1) comienza con las percepciones de deseabilidad —el grado en que la idea de crear una empresa es atractiva (actitud hacia el comportamiento y norma subjetiva) - y de viabilidad — el grado en que uno se cree capaz de ponerla en marcha (control conductual percibido y autoeficacia)-.

\footnotetext{
49 Krueger, N. y Brazeal, D. (1994).

50 Krueger, N. y Brazeal, D. (1994); Shapero, A. y Sokol, L. (1982).

51 Elfving, J.; Brannback, M. y Carsrud, A. (2009).

52 Shapero, A. y Sokol, L. (1982).

53 Ajzen, I. (1991).
} 


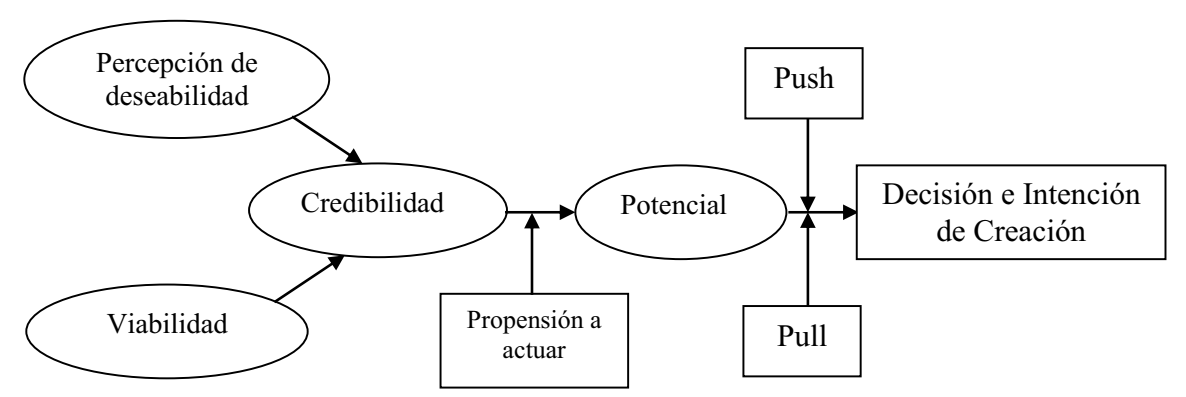

Fuente: Adaptado de Krueger, N. y Brazeal, D. (1994).

Ambos tipos de percepciones son fundamentales para que el comportamiento en cuestión sea creíble ${ }^{54}, \mathrm{y}$ así comenzar a ver este comportamiento con un alto potencial de realización, siempre y cuando el individuo presente también una alta propensión a actuar, una característica de la personalidad más o menos estable, que podría ser fomentada y formada. De hecho, es la combinación de la credibilidad de la idea de emprender con una alta propensión a actuar, esto es, la sensación de ser capaz de iniciar y sostener un comportamiento aún cuando se presenten fuertes adversidades, lo que precipitaría un alto potencial de realización de dicho comportamiento. No obstante, aún cuando la creación de una empresa sea deseable y viable, y por tanto creíble, y con gran potencial de realización, es posible que el individuo no se decida finalmente por crear su empresa. Ello es probable que se deba a la ausencia en él de motivaciones internas (pull), o de factores disparadores (push) ${ }^{55}$.

En efecto, existen una serie de variables pull y push a las que se le concede especial relevancia a la hora de tirar de o empujar al individuo hacia una decisión de creación empresarial ${ }^{56}$. Por un lado, los factores pull hacen referencia

54 Elfving, J.; Brannback, M. y Carsrud, A. (2009).

55 Krueger, N. y Brazeal, D. (1994).

56 Vecciana, J.M. (2005). 
a las motivaciones que le vienen al individuo por crear una empresa. El dinero que se obtiene, el status social que se adquiere, o el deseo de independencia y la necesidad de auto-realización, son algunos de estos factores. Por otro lado, los factores push empujan y precipitan la toma de dicha decisión, si bien éstos tienen ver con cambios relevantes en la situación o estado personal tales como el desempleo de larga duración, un despido reciente, una situación de exclusión social, la falta de seguridad en el empleo y/o la insatisfacción actual en el puesto de trabajo o en la vida, en general.

\subsection{La empresa social y de economía de comunión como oportunidad empresarial}

El fuerte carácter social de este tipo de emprendimiento, orientado a impactar positivamente en la sociedad nos lleva, inevitablemente, a pensar en este tipo de empresas como una fuente importante de generación de proyectos de auto-empleo. De hecho, el desarrollo de modelos económico-empresariales de este tipo suele traer emparejado una superestructura que apoya y favorece la creación de oportunidades para el autoempleo de naturaleza social, a través, principalmente, de asesoramiento y financiación. En efecto, por un lado, este tipo de emprendimiento lleva asociado la existencia de: a) fundaciones/instituciones/asociaciones de apoyo-coordinación ${ }^{57}, \mathrm{~b}$ ) empresas y polígonos industriales de similar naturaleza que actúan como motor de creación de valor y oportunidades empresariales ${ }^{58}$, c) agrupaciones empresariales que crean eventos, reuniones, talleres de trabajo, etc. que favorecen el contacto inter-empresarial, y el intercambio de ideas y apoyos (Business with Social Value, Hub Madrid ${ }^{59}$ ), d) programas de las administraciones públicas (AA.PP.) dedicados a promocionar y financiar este tipo de iniciativas ${ }^{60}$, y e) un número creciente de empresas de capital riesgo orientadas a proveer recursos financieros y consultoría para este tipo de proyectos ${ }^{61}$. Por otro lado, una empresa de este tipo se caracteriza, principalmente, por su cultura de ayudar a la iniciación y desarrollo de otros proyectos emprendedores ${ }^{62}$. En efecto, para Yunus $^{63}$, un fuerte defensor del emprendimiento social, ayudar a los más necesitados pasa inevitablemente por darles la oportunidad de crear su propia

\footnotetext{
57 Linard, K. (2003).

58 Linard, K. (2003); Mair J. y Schoen, O. (2007).

59 http://www.businesswithsocialvalue.org/es; http://madrid.the-hub.net/quienes-somos

60 Comisión Europea (2012b).

61 Certo, S.T. y Miller, T. (2008).

62 Mair, J. y Schoen, O. (2007).

63 Yunus, M. (2008).
} 
empresa, pues es así cuando estas personas se convierten en agentes principales y activos de su propio desarrollo y realización personal y profesional. Además, es típico de este tipo de empresas, en concreto, de las del tipo de economía de comunión, buscar siempre una cultura de reciprocidad auténtica ${ }^{64}$, no basada en el "yo doy" y "tú recibes", sino en el "yo doy, tú recibes y das a la vez" ${ }^{65}$. De esta forma, la cultura de crear valor social no se estanca en la figura de unos pocos, sino que se extiende abiertamente entre otros agentes con ánimo de contribuir a la configuración de un nuevo modelo económico-empresarial más humanamente enriquecedor para la sociedad.

\section{EL PAPEL DE LA MUJER EN EL PROCESO DE DECISIÓN E INTENCIÓN DE CREAR LA EMPRESA SOCIAL O DE ECONOMÍA DE COMUNION}

Estos nuevos enfoques socioeconómicos, que entienden la generación de riqueza inextricablemente unida a la búsqueda/contribución de un impacto social positivo, es probable que se configuren como alternativas óptimas para un perfil determinado de emprendedor. En concreto, la misión de contribuir y tener un impacto positivo en la sociedad parece más afín a un colectivo especialmente sensible en materia social y que se siente descontento con un desarrollo de la actividad económica que genere desigualdades sociales e impacte negativamente en términos sociales. No es extraño, por tanto, que en este tipo de empresas la variable "mujer", mucho más afín a las cualidades sociales anteriormente descritas, se vea altamente representada en la tasa de emprendedores de este tipo de empresas ${ }^{66}$.

Cuando analizamos el emprendimiento empresarial de corte social (empresa social y/o de economía de comunión) frente al emprendimiento puramente comercial, se muestran diferencias significativas entre ambos sexos. En este sentido, la tabla 3 muestra el porcentaje de mujeres frente a hombres que deciden iniciar uno de estos tipos de emprendimiento en varias regiones del mundo (Europa Oriental, Europa Occidental, Australia y Estados Unidos, Latino América y Sudeste Asiático).

\footnotetext{
64 Bruni, L. y Zamagni, S. (2004).

65 Gold, L. (2010).

$66 \operatorname{GEM}(2015)$.
} 
Tabla 3. Influencia del género en el emprendimiento social y comercial

\begin{tabular}{ccccccc}
\hline & $\begin{array}{c}\text { Sudeste } \\
\text { Asiático }\end{array}$ & $\begin{array}{c}\text { Norte } \\
\text { de África }\end{array}$ & $\begin{array}{c}\text { Europa } \\
\text { Oriental }\end{array}$ & $\begin{array}{c}\text { Europa } \\
\text { Occidental }\end{array}$ & $\begin{array}{c}\text { Australia y Estados } \\
\text { Unidos }\end{array}$ & $\begin{array}{c}\text { Latino } \\
\text { América }\end{array}$ \\
$\begin{array}{c}\text { Emprendimiento } \\
\text { Social } \\
(\% \text { mujeres })\end{array}$ & $44 \%$ & $50 \%$ & $46 \%$ & $42 \%$ & $48 \%$ & $45 \%$ \\
$\begin{array}{c}\text { Emprendimiento } \\
\text { Comercial } \\
(\% \text { mujeres })\end{array}$ & $45 \%$ & $37 \%$ & $36 \%$ & $35 \%$ & $36 \%$ & $46 \%$ \\
\hline
\end{tabular}

Fuente: Elaboración propia a partir de GEM (2015).

En la tabla 3 podemos observar que, en muchas regiones, a excepción de la correspondiente a las economías latinoamericana ( $46 \%$ de mujeres frente a un $54 \%$ de hombres) y del sudeste asiático (45\% de mujeres frente a un 55\% de hombres), los empresarios comerciales varones superan en número a las mujeres, aproximadamente como dos a uno. Por ejemplo, en Europa oriental y occidental, las mujeres empresarias comerciales comprenden solo el $36 \%$ y el $35 \%$ de todos los empresarios, respectivamente. Sin embargo, en el caso del emprendimiento social (empresa social y/o de economía de comunión), la brecha es menos pronunciada, obteniéndose prácticamente la paridad en todas las regiones analizadas, e incluso llegándose a conseguir ésta en la región del Norte de África.

En efecto, la mujer tiene en este modelo económico-empresarial una solución a su mayor interés por impactar positivamente en la sociedad, principalmente, a través de la creación de su propia empresa. Por un lado, las mujeres se suelen caracterizar por percibir y entender sus vidas en términos de tener una misión y un compromiso de servicio, en clara contraposición a la obtención de beneficios y ganancias financieras ${ }^{67}$; es más, las mujeres, por lo general, se caracterizan por tener una mayor sensibilidad a las necesidades de terceras personas, y a los problemas sociales en general ${ }^{68}$. De hecho, numerosos estudios revelan una mayor orientación por parte de las mujeres a proyectos de naturaleza social ${ }^{69}$, y a una mayor predisposición a actuar en favor de la

67 Fisher, H. (1999).

68 McGowan, P.; Cooper, S.; Durkin, M. y O'Kaine, C. (2015).

69 Cukier, W.; Trenholm, S.; Carl, D. y Gekas, G. (2011); Witkamp, M.; Royakkers, L. y Raven, R. (2011). 
comunidad, ayudando y construyendo para otras personas ${ }^{70}$. Tal disposición a resolver problemas sociales se identifica con la misión fundacional de este tipo de empresas ${ }^{71}$. Además, la mujer tiene mayor predisposición por fomentar la justicia y la responsabilidad ${ }^{72}$, mayor capacidad de conocer e identificar lo que es ético o no, y mayor predisposición a comportarse éticamente bien ${ }^{73}$, lo que se encasilla perfectamente con la naturaleza de los valores de quienes emprenden este tipo de empresas ${ }^{74}$. Por todo ello, es de esperar que, para las mujeres, la idea de crear una empresa de marcado acento social sea altamente atractiva; mediante la creación de este tipo de empresas es más probable que este colectivo realice su vocación de servicio y alcance su plenitud personal, lo que actuaría como factor pull en la creación de este tipo de empresas (Figura 2).

Por otro lado, el colectivo de la mujer representa un colectivo que, a priori, juega en desventaja en materia de acceso al mercado laboral y empresarial $^{75}$, y de promoción en la carrera profesional ${ }^{76}$, y se le asocia así con un mayor riesgo de exclusión social ${ }^{77}$. De hecho, dicho colectivo tiende a ser la parte de la relación de pareja que sufre con más virulencia e intensidad el fenómeno de la violencia de género, lo que supone un detonante para llegar, más fácilmente, a una situación de riesgo de exclusión socia ${ }^{78}$. Estos factores, junto a otros más generales (p.e. ser objeto de despido), actuarían como factores push que empujarían o dispararían a este colectivo a crear una empresa de marcado acento social (Figura 2).

70 Humbert, A. L. (2012); Cukier, W.; Trenholm, S.; Carl, D. y Gekas, G. (2011).

71 Seelos, C. y Mair, J. (2005); André, K. y Pache, A. C. (2016).

72 Libby, B. y Agnello, V. (2000).

73 Collins, D. (2000).

74 Bruni L. y Zamagni, S. (2004); Humbert, A. L. (2012); Seelos, C. y Mair, J. (2005).

75 De la Fuente, R. y González-Castro, J. L. (2009); Justo, R. y García, C. (2012).

76 WISE (2015).

77 Subirats, J. (2004).

78 De la Fuente, R. y González-Castro, J. L. (2009). 
Figura 2. Mujer y creación de "empresas sociales y/o de economía de comunión”

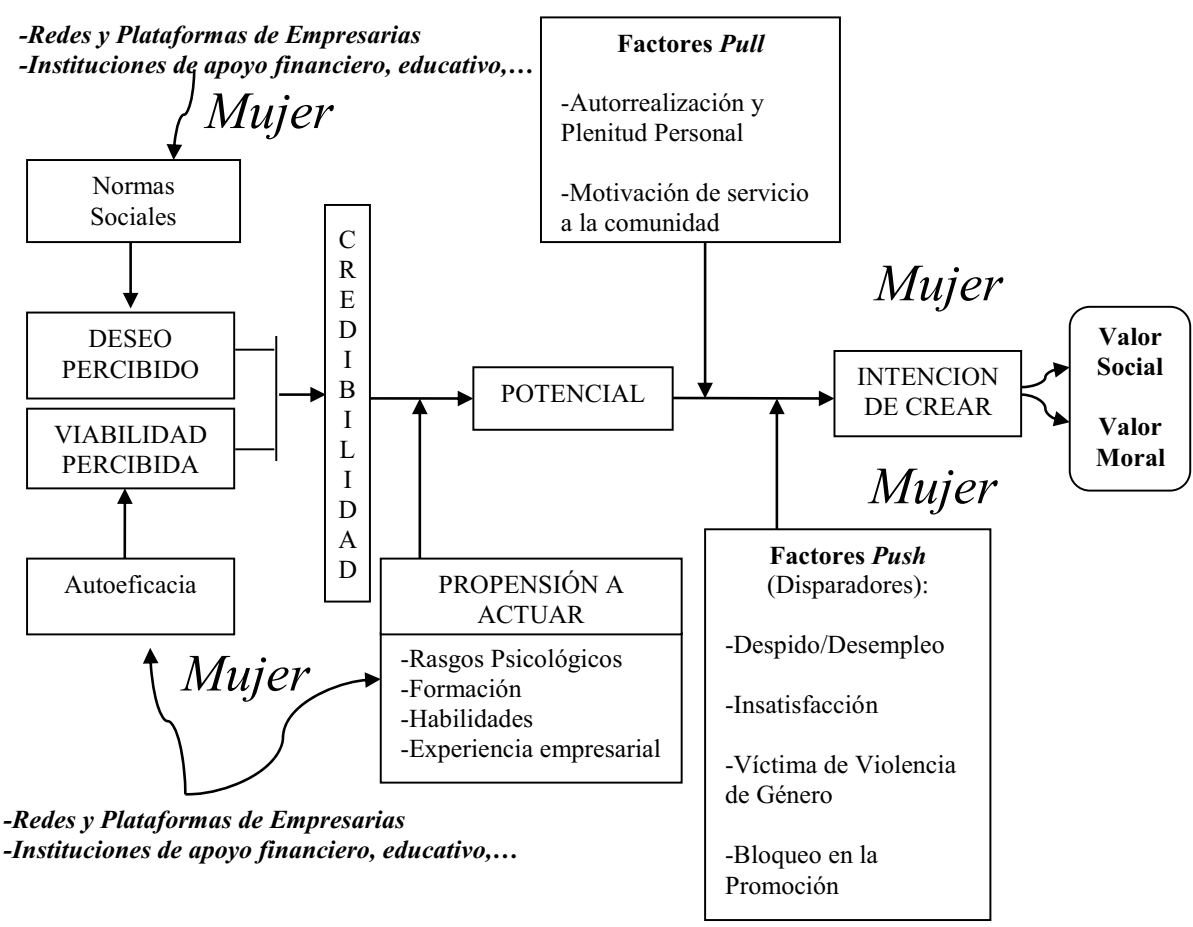

Fuente: Elaboración propia a partir de Krueger, N. y Brazeal, D. (1994).

No obstante lo anterior, existen además una serie de factores que van a influir tanto a) en la deseabilidad y viabilidad percibida por parte de la mujer, esto es, en la credibilidad que tiene la acción de crear una empresa, como también b) en la propensión efectiva a crearla ${ }^{79}$ (ver Figura 2). Todos estos factores son los que, de manera previa a los factores pull y push, afectarán la decisión de la mujer de crear una empresa, a través de determinar el grado de potencial empresarial de dicha idea. Así, con respecto a la deseabilidad percibida, la influencia de los factores culturales, políticos, sociales y macroeconómicos puede tomar un papel ciertamente cambiante y diferencial, depen-

79 Krueger, N. y Brazeal, D. (1994). 
diendo del sexo del individuo. En efecto, a la variable "sexo" se le suele atribuir una influencia importante en la forma de percibir la realidad y decidir las actitudes y comportamientos a desarrollar ante las adversidades ${ }^{80}$. De hecho, las normas sociales, según se contemplan en la actualidad, a priori, pueden limitar y desanimar al colectivo de la mujer de su deseo de crear una empresa, pues la idea de que el empresario de éxito suele tener características masculinas se encuentra muy extendida en la sociedad, incluso, entre las propias mujeres ${ }^{81}$. Como resultado, es probable que las percepciones que tienen los propios agentes socio-económicos sobre el éxito operativo de cualquier actividad empresarial iniciada y desarrollada por mujeres sean negativas, limitando así, la movilización de recursos hacia éstas y su capacidad de generación de negocio. Sin embargo, en el caso de la creación de una empresa de marcado acento social, las mujeres disponen de múltiples recursos de relaciones, financiación y asesoramiento que actúan como fuerza facilitadora del desarrollo de la actividad empresarial, aumentando la deseabilidad social percibida de la creación empresarial. En efecto, es creciente el número de plataformas, fundaciones y asociaciones constituidas en torno a la figura de un emprendedor que desea impactar positivamente sobre la sociedad, y en particular, al colectivo de la mujer emprendedora ${ }^{82}$, con el firme objetivo de facilitar contactos, apoyo y asesoramiento. Todo ello supone para este colectivo una fuente continua de apoyo y energía con la que percibir una alta deseabilidad social sobre la idea de crear este tipo de empresas.

Por su parte, en relación a la viabilidad percibida de la idea de creación, esto es, el grado en que la mujer se cree capacitada para la creación de una empresa, ésta se encuentra estrechamente relacionada con su grado de autoeficacia. La autoeficacia se entiende como "los juicios que cada persona tiene sobre sus capacidades, con base en las cuales organizará y ejecutará sus actos de modo que le permitan alcanzar el rendimiento deseado" ${ }^{\prime 3}$. Esto es, la autoeficacia es la confianza de la persona sobre sus capacidades para obtener los resultados anhelados, de tal forma que las capacidades reales sólo importarían si la persona tiene confianza en las mismas. No es extraño, por tanto, que este factor, a través de aumentar la percepción de capacidad de crear con éxito una empresa, influya positivamente sobre la percepción de viabilidad de esta idea. Es verdad que las mujeres parecen mostrar una menor autoeficacia y una menor confianza en

\footnotetext{
80 Greer, M. y Greene, P. G. (2003).

81 Ching-Yin Yim, P. y Harris, M. (2002).

82 Spark Her, Young Women Social Entrepreneurs, Ladies who Launch, y Mom Invented son algunas de ellas. 83 Bandura, A. (1986).
} 
sus capacidades para obtener determinados resultados ${ }^{84}$, especialmente si éstas tienen que ver con capacidades típicas "masculinas" relacionadas con la actividad y creación empresarial ${ }^{85}$. Además, las mujeres, por lo general, están menos predispuestas a enfrentar desafíos/retos, e iniciar proyectos empresaria1 les ${ }^{86}$, presentando un mayor temor al fracaso ${ }^{87}$, y en consecuencia, una percepción más negativa sobre la viabilidad de la idea de crear una empresa. Sin embargo, de la mano de asociaciones, fundaciones, e instituciones centradas en apoyar, acompañar, formar y asesorar en materia de creación de empresas con marcado acento social, las mujeres pueden encontrar mucho más viable la posibilidad de crear proyectos de este tipo. En efecto, la formación en materia de emprendimiento es importante a la hora de influir positivamente sobre el nivel de autoeficacia en mujeres ${ }^{88}$, y ésta se ofrece comúnmente en las entidades anteriormente mencionadas, lo que podría influir positivamente sobre la percepción que tienen las mujeres en materia de viabilidad de la idea de creación de una empresa de marcado acento social.

Finalmente, una vez que la idea de creación es tanto necesaria como viable, y en consecuencia, genera credibilidad, resultaría necesaria cierta propensión a la creación para alcanzar un alto potencial de creación. Esta propensión a crear empresas podría alimentarse, principalmente, de factores relacionados a) con la formación del individuo, b) con la experiencia laboral y c) con determinados factores psicológicos, entre otros ${ }^{89}$. En este sentido, y aunque todavía no se ha podido esclarecer el perfil psicológico único que determinaría la propensión a la creación de empresas, la literatura ha podido establecer una lista de algunos de ellos como ciertamente influyentes. Entre ellos, nos encontramos con la creatividad, la capacidad de innovación y la capacidad de aceptación de responsabilidades ${ }^{90}$, aspectos éstos, que en el contexto de creación de empresas con marcado acento social, juegan un papel ciertamente importante ${ }^{91}$. En este sentido, la literatura sugiere que "ser mujer" se ajusta perfectamente a la posesión de estos rasgos, puntuando de manera elevada en apertura a los sentimientos ${ }^{92}$-íntimamente relacionada con la cre-

84 Wilson, F.; Kickul, J. y Marlin, D. (2007).

85 Marlin, D. y Wilson, F. (2003).

86 Langowitz, N. y Minniti, M. (2007).

87 Justo, R. y García, C. (2012).

88 Wilson, F.; Kickul, J. y Marlin, D. (2007).

89 Shane, S. (2003).

90 McClelland, D. (1961).

91 Nga, J. K. H. y Shamuganathan, G. (2010).

92 Costa, P. T.; Terracciano, A. y McCrae, R. R. (2001). 
atividad y la curiosidad-, y en responsabilidad, y amabilidad ${ }^{93}$, rasgos que además, hacen más probable la intención emprendedora de naturaleza social ${ }^{94}$. Por otro lado, tanto en lo que respecta a la formación en materia de emprendimiento como a la experiencia laboral, éstos son aspectos que, de manera creciente, se favorecen en el contexto de este tipo de emprendimiento. Como ya se adelantó, las mujeres que emprenden con un marcado acento social cuentan con un amplio abanico de asociaciones, fundaciones, plataformas, e instituciones cuya existencia, además de suponer un punto de encuentro y de intercambio de experiencias entre empresarios, es fuente de apoyo, formación $\mathrm{y}$ asesoramiento en materia de emprendimiento.

\section{CONCLUSIONES}

La sensibilidad social por parte de inversores, grupos de interés y sociedad en general es cada vez mayor. Los requerimientos de éstos por un mundo económico-empresarial más justo y preocupado por su rendimiento social son cada vez mayores ${ }^{95}$, suponiendo así un importante germen para el desarrollo de modelos socioeconómicos alternativos, más justos y con un enfoque social. En concreto, son la red del emprendimiento social y la economía de comunión los enfoques que han emergido con fuerza durante las últimas décadas, en su afán de lograr un modelo económico de "aldea global" basado en los principios de "redistribuir", "donar" e "intercambiar", entre y para todos los agentes de la sociedad ${ }^{96}$. Así, el tipo de empresarios que lo secunda suele responder a la necesidad de abordar desigualdades y problemas sociales existentes, en parte derivados de la operativa del sistema capitalista actual.

El factor "mujer" parece desempeñar un papel determinante sobre la decisión e intención de crear y poner en marcha un proyecto de emprendimiento social o de economía de comunión. En concreto, del análisis efectuado se puede concluir que, en un contexto socio-económico como el actual, de crecimiento y apoyo a este modelo socioeconómico ${ }^{97}$, las mujeres representan un colectivo afín a la creación de este tipo de empresas, cuya interacción "mujeremprendimiento social/economía de comunión" favorece todos los elemen-

93 Schmitt, D. P.; Realo, A; Voracek, M. y Allik, J. (2008).

94 Nga, J. K. H. y Shamuganathan, G. (2010).

95 Carroll, A. B. y Bucholtz, A.K. (2008).

96 Bruni, L. y Zamagni, S. (2004).

97 Comisión Europea, (2012b); Gold, L. (2004); Gold, L. (2010). 
tos y fases determinantes en la decisión de crear una empresa. En efecto, la propia naturaleza y características personales de este colectivo hacen de este modelo socioeconómico - un modelo híbrido surgido en el tercer sector que desdibuja/difumina los límites existentes entre los tres sectores de la economía: Privado, Público y No Lucrativo ${ }^{98}$ - , uno óptimo para que el colectivo de la mujer se desarrolle profesionalmente ${ }^{99}$, a través de iniciar una actividad empresarial. Simplemente, su menor orientación a medir el éxito de una empresa a través de criterios meramente cuantitativos ${ }^{100}$ es un aspecto esclarecedor sobre la idoneidad de este colectivo a la hora de iniciar proyectos de este tipo. La interacción "mujer-emprendimiento social/economía de comunión" parece facilitar el cumplimiento de todas las fases necesarias para la puesta en marcha de una empresa, en concreto, de este tipo de empresas.

Así, del análisis realizado se concluye que existe la necesidad de promover desde las AA.PP. la creación de este tipo de empresas, donde sus responsables no deberían obviar el diseño y desarrollo de determinadas líneas estratégicas de acción, centradas en promover el empresariado social y poner en contacto a este colectivo con instituciones, asociaciones, o fundaciones creadas en torno a este concepto de empresa. En efecto, un esfuerzo por diseñar políticas que se diseñen en torno a promover el empresariado social y de economía de comunión entre el colectivo de la mujer, y en concreto, a poner en contacto a este colectivo con los agentes, asociaciones y fundaciones encargadas de promover esta iniciativa empresarial, es muy probable que contribuya de manera efectiva a impulsar este tipo de emprendimiento, que como se refleja en la figura anterior (ver Figura 2), representa una fuente importante de valor e innovación social y moral ${ }^{101}$.

98 Defourny, J. y Nyssens, M. (2012).

99 Mouazan, S. (2012).

100 Cliff, J. (1998).

101 Crivelli, L. y Gui, B. (2014); Kuratko, D. F. (2009). 


\section{BIBLIOGRAFÍA}

André, Kevin y Pache, Anne C. (2016), "From Caring Entrepreneur to Caring Enterprise: Addressing the Ethical Challenges of Scaling up Social Enterprises", Journal of Business Ethics, vol. 133, n 4, pp. 659-675.

Azjen, Icek (1991), "The Theory of Planned Behavior". Organizational Behavior and Human Decision Processes, vol. 50, pp. 179-211.

Bandura, Albert (1986), Social Foundations of Thought and Action: A Social Cognitive Theory, Prentice Hall, Englewood Cliffs.

Braga, Joao; Proença, Teresa y Ferreira, Marisa (2014), "Motivations for Social Entrepreneurship-Evidences from Portugal", Tekbne, vol. 13, pp. 1121.

Bruni, Luigino (2002), The Economy of Communion-Toward a Multi-Dimensional Economic Culture, New City Press, Hyde Park.

Bruni, Luigino y Zamagni, Stefano (2004), "The Economy of Communion: Inspirations and Achievements", Finance \& The Common Good, vol. 20, pp. 91-97.

Bureau of European Policy Advisers (2010), Empowering People, Driving Change, Social Innovation in the European Union, EUR-OP, Luxembourg.

Carroll, Archie B. y Bucholtz, Ann K. (2008), Business and Society: Ethics and Stakeholder Management, South Western Cengage Learning, Ohio.

Certo, Trevis, S. y Miller, Toyah (2008), "Social Entrepreneurship: Key Issues and Concepts”, Business Horizons, vol. 51, pp. 267-271.

Ching-Yin Yim, Phillis y Harris, Michael (2002), "Gender Stereotyping of Managers and self-Concept of Business Students across their Undergraduate Education", Women in Management Review, vol. 17, pp. 364-373.

Cliff, Jennifer (1998), "Does one Size fit All? Exploring the Relationship between Attitudes towards Growth, Gender, and Business Size", fournal of Business Venturing, vol. 13, pp. 523-542.

Collins, Denis (2000), "The Quest to Improve the Human Condition", Fournal of Business Ethics, vol. 26, pp. 1-73. 
Comisión Europea (2012a), The Social Business Initiative of the European Commission. Recuperado de http://ec.europa.eu/internal_market/publications (Consultado el 20 de febrero de 2016).

Comisión Europea (2012b), Propuesta de Resolución sobre la Iniciativa a favor del emprendimiento social: Construir un ecosistema para promover las empresas sociales en el centro de la economía y la innovación sociales. Consultado de http://www.europarl.europa.eu/ (Consultado el 25 de febrero de 2016).

Comisión Europea (2015), Policy Brief on Social Impact Measurement for Social Enterprises, Recuperado de http://ec.europa.eu/social/ (Consultado el 25 mayo de 2016).

Costa, Paul T. Jr.; Terracciano, Antonio y McCrae, Robert R. (2001), "Gender Differences in Personality Traits across Cultures: Robust and Surprising Findings", Fournal of Personality and Social Psychology, vol. 81, pp. 322331.

Crivelli, Luca y Gui, Benedetto (2014), "Do Economy of Communion Enterprises Deserve the Social Label? A Comparative Discussion of their Aims and Logic of Action", Revista Portuguesa de Filosofía, vol. 70, pp. 28-43.

Cukier, Wendy; Trenholm, Susan; Carl, Dale y Gekas, George (2011), "Social Entrepreneurship: A Content Analysis", Fournal of Strategic Innovation and Sustainability, vol. 7, n ${ }^{\circ}$ 1, pp. 99-119.

De la Fuente, Raquel y González-Castro, José Luis (2009), "Empleo, Formación e Inserción de Colectivos en Riesgo de Exclusión. Un Reto Social y Económico para la Empresa Receptora”, Bordón, vol. 61, pp. 33-46.

Dees, J. Gregory (2011), "El significado del emprendimiento social”, $R e-$ vista Española del Tercer Sector, vol. 17, pp. 111-124.

Defourny, Jácques y Nyssens, Marthe (2008), “Social Enterprise in Europe: Recent Trends and Developments", Social Enterprise fournal, vol. 4, pp. 202-228.

Defourny, Jácques y Nyssens, Marthe (2012), “El Enfoque EMES de la Empresa Social desde una perspectiva comparada", CIRIEC-España, Revista de Economía Pública, Social y Cooperativa, vol. 75, pp. 7-34.

Economy of Communion (2016), EoC Business Guidelines, Recuperado de http://www.edc-online.org/en/businesses/guidelines-for-conducting-a-business.html (Consultado el 9 de julio de 2016). 
Elfving, Jennie; Brannback, Malin y Carsrud, Alan (2009), “Toward a Contextual Model of Entrepreneurial Intentions", en Carsrud, Alan y Brannback, Malin (eds.), Understanding the Entrepreneurial Mind, Springer, New York.

Enciso, Marta, Gómez, Laura y Mugarra, Aitziber (2012), "La iniciativa comunitaria a favor del emprendimiento social y su vinculación con la economía social: una aproximación a su delimitación conceptual”, CIRIEC-España, Revista de Economía Pública, Social y Cooperativa, vol. 75, pp. 55-80.

EUROSTAT (2012), 23\% of EU citizens were at risk of poverty or social exclusion in 2010. European Union, Belgium. Recuperado de http://ec.europa.eu/eurostat/en/web/products-statistics-in-focus/-/KS-SF-12-009 (Consultado el 15 de mayo de 2016).

EUROSTAT (2014), Living conditions in Europe, 2014 Edition. European Union, Belgium. Recuperado de http://ec.europa.eu/eurostat/web/productspress-releases/-/3-17102016-BP (Consultado el 14 de mayo de 2016).

EUROSTAT (2016), The Share of Persons at Risk of Poverty or Social Exclusion in the EU back to its pre-crisis Level. European Union, Belgium. Recuperado de http://ec.europa.eu/eurostat/web/products-statistical-books/-/KSDZ-14-001 (Consultado el 10 de diciembre de 2017).

Fisher, Helen (1999), The First Sex: The Natural Talents of Women and how they are Changing the World, Ballantine Books, New York.

GEM (2016), The 2016/2017 Global Report. Recuperado de http://www.gemconsortium.org/report (Consultado el 14 julio de 2017).

GEM (2015), Special Topic Report Social Entrepreneurship. Recuperado de http://gemconsortium.org/report/49542 (Consultado el 12 de julio de 2016).

Gold, Lori (2004), The Sharing Economy, Solidarity Networks Transforming Globalisation, Ashgate Publishing Limited, Burlington.

Gold, Lori (2010), New Financial Horizons, the Emergence of an Economy of Communion, New City Press, Hyde Park.

Greer, Margaret. J. y Greene, Patricia G. (2003), "Feminist Theory and the Study of Entrepreneurship", en Butler, John (ed.), New Perspectives on Women Entrepreneurs, Information Age Publishing, Connecticut.

Grochmal, Stanislaw (2016), "Spirituality of Unity in Management-Economy of Communion”, Cogent Business \& Management, vol. 3, n 1, pp.1-18. 
Humbert, Anne L. (2012), "Women as Social Entrepreneurs”, Working Paper, $\mathrm{n}^{\circ}$ 72. Third Sector Research Centre, Park House, Birmingham.

Justo, Rachida y García, Cristina (2012), "Incidencia de los Modelos de Referencia en la Creación de Empresas. Efectos Mediadores y de Género", Economía Industrial, vol. 383, pp. 111-124.

Krueger, Norris y Brazeal, Deborah (1994), "Entrepreneurial Potential and Potential Entrepreneurs", Entrepreneurship Theory and Practice, vol. 18, pp. 91-104.

Krugman, Paul (2008), The Return of Depression Economics and the Crisis of 2008, W.W. Norton \& Company, Londres.

Kuratko, Donald F. (2009), Entrepreneurship: Theory, Process Practice, South-Western Cengage Learning, Mason.

Langowitz, Nan y Minniti, Maria (2007), “The Entrepreneurial Propensity of Women", Entrepreneurship Theory and Practice, vol. 31, pp. 341-364.

Libby, Barbara y Agnello, Vincent (2000), "Ethical Decision Making and the Law", Fournal of Business Ethics, vol. 26, pp. 223-232.

Linard, Keith (2003), "Economy of Communion: Systemic Factors in the Rise of a New Entrepreneurship", Systems Research \& Behavioral Science, vol. 20, pp. 163-175.

López, Katherine J.; Martínez, Zaida L. y Specht, Linda B. (2013), “The Economy of Communion Model: A Spirituality-Based View of Global Sustainability and its Application to Management Education", Fournal of Management for Global Sustainability, vol. 1, pp. 71-90.

Lubich, Chiara (2003), Economía de Comunión. Historia y Profecía, Ciudad Nueva Grupo Editorial, Madrid.

Mair, Johanna y Schoen, Oliver (2007), "Successful Social Entrepreneurial Business Models in the Context of Developing Economies: An Explorative Study", International Fournal of Emerging Markets, vol. 2, pp. 54-68.

Marlino, Deborah y Wilson, Fionna (2003), Teen Girls on Business: Are They Being Empowered?, Simmons School of Management and The Committee of 200, Boston.

Martin, Roger L. y Osberg, Sally (2007), "Social Entrepreneurship: The Case for Definition”, Stanford Social Innovation Review, vol. 5, pp. 28-39. 
Martinelli, Flavia (2012), "Social Innovation or Social Exclusion? Innovating Social Services in the Context of a Retrenching Welfare State", en Franz, Hans W.; Hochgerner, Josef y Howaldt, Jürgen (eds.), Challenge Social Innovation, Springer, Berlin, pp. 169-180.

McClelland, David (1961), The Achieving Society, D. Van Norstrand Co, Princeton.

McGowan, Pauric; Cooper, Sarah; Durkin, Mark y O'Kane, Caroline (2015), "The Influence of Social and Human Capital in Developing Young Women as Entrepreneurial Business Leaders", Journal of Small Business Management, vol. 53, $\mathrm{n}^{\circ} 3$, pp. 645-661.

Mouazan, Servane (2012), "The Secret Algorithm of Women Social Innovators", The Guardian, Recuperado de https://socialenterprise. guardian.co.uk (Consultado el 20 de mayo de 2016).

Mulgan, Geoff; Tucker, Simon; Ali Rushanara y Sanders, Ben (2007), Social Innovation, what it is, why it Matters, and how it can be Accelerated, The Basingstoke Press, Londres.

Nga, Joyce K.H. y Shamuganathan, Gomathi (2010), “The Influence of Personality Traits and Demographic Factors on Social Entrepreneurship Start Up Intentions", Fournal of Business Ethics, vol. 95, pp. 259-282.

Pol, Eduardo y Ville, Simon (2009), "Social Innovation: Buzz Word or Enduring Term”, The Fournal of Socio-Economics, vol. 38, pp. 878-885.

Perrini, Francesco y Vurro, Clodia (2006), "Social Entrepreneurship: Innovation and Social Change across Theory and Practice", en Mair, Johanna, Robinson, Jeffrey y Hockerts Kai (eds.), Social Entrepreneurship, Palgrave Macmillan, Londres.

Schmitt, David P.; Realo, Anu; Voracek, Martin y Allik, Juri (2008), "Why can't a Man be more like a Woman? Sex differences in Big Five Personality Traits across 55 Cultures", fournal of Personality and Social Psychology, vol. 94, pp.168-182.

Seelos, Christian y Mair, Johanna (2005), "Social Entrepreneurship: Creating new Business Models to Serve the Poor", Business Horizons, vol. 48, pp. 241-246.

Sen, Amartya (2010), "Adam Smith and the Contemporary World", Erasmus Fournal for Philosophy and Economics, vol. 3, pp. 50-67. 
Shane, Scott (2003), A General Theory of Entrepreneurship-The Individual Oportunity Nexus, Edward Elgar, Northampton.

Shapero, Albert y Sokol, Lisa (1982), "The Social Dimensions of Entrepreneurship”, en Kent, Calvin; Sexton, Donald y Vesper, Karl H. (eds.), The Encyclopedia of Entrepreneurship, Prentice Hall, Englewood Cliffs, pp. 72-90.

Smith, Adam [1776(2009)], Una Investigación sobre la Naturaleza y Causa de la Riqueza de las Naciones, Tecnos, Madrid.

Smith, Adam [1759 (2004)], La Teoría de los Sentimientos Morales, Fondo de Cultura Económica, México.

Subirats, Joan (2004), Pobreza y exclusión social. Un Análisis de la Realidad Española y Europea, Colección Estudios Sociales, Fundación La Caixa, Barcelona.

Vecciana, José María (2005), La Creación de Empresas, un enfoque general. Colección Estudios Económicos, Fundación La Caixa, Barcelona.

Wilson, Fiona; Kickul, Jill y Marlin, Deborah (2007), "Entrepreneurship Gender, Entrepreneurial Self-Efficacy and Entrepreneurial Career Intentions: Implications for Entrepreneurshhip Education", Entrepreneurship Theory and Practice, vol. 31, pp. 387-406.

WISE (2015), Proyecto Women Innovators for Social Business in Europe. Recuperado de http://wise4women.eu/countries/spain. (Consultado el 14 Julio de 2017).

Witkamp, Marten; Royakkers, Lamber y Raven, Rob (2011), "From Cowboys to Diplomats: Challenges for Social Entrepreneurship in the Netherlands", Voluntas, vol. 22, pp. 283-310.

Yunus, Muhammad (2008), El banquero de los pobres: los microcréditos y la batalla contra la pobreza en el mundo, Editorial Paidós Ibérica, Barcelona. 

RECENSIONES 
